\title{
Radar Downstream Channel Phase Noise Measurement and Analysis
}

\author{
Rui $X u^{1, a^{*}}$, Jianhong $X u^{2, b}$ and Xinfeng $\operatorname{Fan}^{3, c}$ \\ ${ }^{1,2,3}$ China Satellite Maritime Tracking and Control, Jiangyin, Jiangsu, China \\ axurui080267@163.com, bxiaotian86926@163.com, cfanxf0510@163.com
}

Keywords: phase noise, local oscillator, radar receiver.

\begin{abstract}
The channel system parameter of phase noise has an important influence on the performance of the radar receiver. With a brief introduction to the concept and formation mechanism of phase noise, the research was focused on local oscillator phase noise, which has the greatest impact on the performance of the receiver. And the analysis conclusion was finally proved by the actual measurement result of phase noise in a radar downward channel.
\end{abstract}

\section{Introduction}

In the radar receiver system, the radio frequency signal received by the antenna is usually converted to an intermediate frequency by down-conversion one or several times before processed. In this process, the input signal will be distorted by the mixer and local oscillator in the channel, thereby reducing the demodulation performance of the receiver. The impact of mixing noise which introduced into the system by the mixer can be reduced by improving the design of other parts, but the random phase jitter of the local oscillator signal (i.e. phase noise) is difficult to eliminate.

With the limits to the signal-noise ratio and sensitivity of the receiver, phase noise becomes a major factor influencing the performance of the radar system, and its test and analysis has a great significance in the research to improve equipment performance.

\section{Phase Noise}

Phase noise is an important technical indicator to measure the smooth quality of signal, used to characterize the short-term frequency stability.

Typically, the phase of the output signal changes randomly at the excitation of the system internal noise, and the change in the phase will also cause the change in the frequency of the output signal. Frequency stability is used to describe that change in the frequency of the signal within a certain time, divided into long-term stability and short-term stability depending on the length of the interval.

Short-term stability performs as the jitter at the zero point of the waveform in the time-domain, usually expressed by the relative value of changes in the frequency at a certain time interval (Allan variance). Phase noise is its representation in the frequency domain, defined as the noise power ratio of the carrier signal power in a $1 \mathrm{~Hz}$ bandwidth at a given frequency offset. In the actual measurement, it is commonly represented by the phase noise of a single sideband (SSB). [1, 2, 3]

$$
L\left(f_{m}\right)=\frac{P_{S S B}}{P_{S}}=\frac{\text { Power density }(A \text { phase modulation sideband } 1 \mathrm{~Hz})}{\text { The total carrier power }}
$$


The $\mathrm{L}\left(\mathrm{f}_{\mathrm{m}}\right)$ is usually expressed by the logarithm of $1 \mathrm{~Hz}$ bandwidth to the carriers, at the unit of $\mathrm{dBc} / \mathrm{Hz}$.

\section{Impact of Phase Noise at the Performance of the Receiver [4].}

Common configuration of the receiver downlink channel is shown as Fig.1.

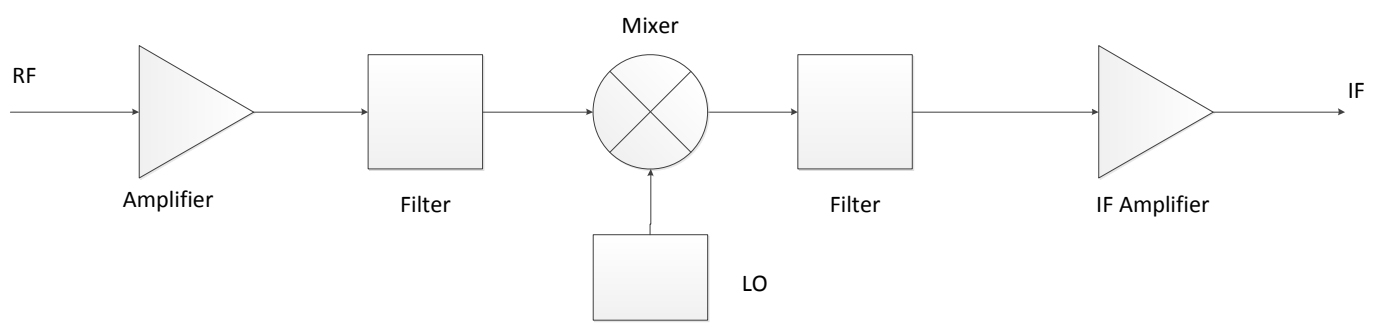

Figure1 the downlink signal processes in the receiver

Assuming the form of an ideal signal as shown in Eq.2.

$$
V(t)=V_{0} \cos \left(t+\theta_{0}\right)
$$

In the Eq.2, $\mathrm{V}_{0}$ and $\theta_{0}$ are all constants, and the signal period is a constant value, the representation in frequency spectrum is a spectrum line. Due to the noise, there are parasitic amplitude modulations and parasitic phase modulations exist in the actual received RF signal, which can be expressed as:

$$
V(t)=V_{0}[1+\alpha(t)] \cos \left[\omega_{0} t+\theta(t)\right]
$$

In the Eq.3, $\alpha$ signifies the random amplitude modulation, and $\theta$ is for the random phase modulation.

In general, $\alpha$ is relatively small, it will not lead to a direct result of the fluctuation in frequency or phase fluctuations, and so it can not affect the frequency stability. Furthermore, since the mixers typically operate at a saturation input power, which reduces its sensibility to amplitude variation, and thus the amplitude noise has little effect on the performance of the receiver, which is negligible.

So $\theta(t)$ is the main factor affecting the spectral purity.

And the Eq.3 can be simplified as Eq.4.

$$
V(t)=V_{0} \cos \left[\omega_{0} t+\theta(t)\right]
$$

In the Eq.4, $\theta(t)=\sin p t, \theta$ signifies the peak angle offset in rad, and $\mathrm{p}$ is a frequency range.

After reorganization the final approximation of phase noise is expressed as Eq.5.

$$
V_{(t)}^{*}=V_{0}\left[\frac{\theta}{2} \sin \left(\omega_{0}+p\right) t-\frac{\theta}{2} \sin \left(\omega_{0}-p\right) t\right]
$$

The input signal $f_{(t)}$ is modulated by the channel phase noise, resulting in impact of phase noise on signal spectrum is:

$$
Y_{(\omega)}=\frac{\theta}{2} V_{0} \pi i\left[F\left(\omega-\omega_{0}+p\right)-F\left(\omega-\omega_{0}-p\right)\right]
$$

Thus, phase noise distributes near the center frequency spectrum of the down conversion signal, which affects the quality of the signal.

All components of the channels will cause phase noise, but in fact when the RF signals pass through the channel, there are few phase noises produced by amplifiers or filters, and the local 
oscillator is the most important source of noise. The following will be mainly about analysis on the local oscillator phase noise. [5]

\section{Oscillator Phase Noise Impact on the Performance of the Receiver [6].}

RF input signal is multiplied by the local oscillator signal in the mixer, and then the desired intermediate frequency signal will be selected by the IF filtering. In this process, the local oscillator phase noise will be "transferred" to the RF signal, appeared as the modulation of the carrier phase. Phase noise constitutes an additional noise to the signal demodulator, and its influence extent depends on the type of modulation.

Restrictions on SNR of FM. LO phase noise will limit the maximum signal to noise ratio which a FM receiver can achieve.

In FM systems, local oscillator phase noise is transferred to the carrier which is to be tuned by the receiver, and then demodulated by a FM discriminator. LO phase noise will produce a fixed noise level at the output of the receiver, if the input level of the receiver is large enough, this will be the dominant noise source, thus limiting the maximum signal to noise ratio that a receiver is able to achieve.

Restrictions on SNR of PM. LO phase noise also limits the SNR of a phase modulation receiver.

Phase modulation is often used to transmit digital signals. For digital signals, carrier phase changes with an integer times of the minimum step size of the phase. LO phase noise will affect the error rate of the phase-shift keying digital transmission systems, when the phase caused by the oscillation noise is large enough, it will lead to mistakes in digital phase detector, resulting in code errors. The smaller the digital transmission system phase stepper is, the more sensitive the effects of local oscillator phase noise will be.

Reciprocal Mixing. When there are strong interferences near the reception frequency, mutual mixing effect will reduce the sensitivity of the receiver.

LO phase noise will be modulated onto the carrier of an interference signal, causing the power spectral density be proportional to the SSB phase noise of the local oscillator. When the receiver is tuned to the close to a strong carrier frequency, power density of the strong carrier noise sidebands will exceed the noise floor of the receiver. If it does not exceed the noise floor, then the sensitivity of the receiver will be limited due to mixing with each other.

In addition to the phase noise, some phase or amplitude modulation clutters are also introduced into by local oscillator. The reasons for these clutters include frequency interference, switching power supply clutters, reference frequency clutters, or other oscillators and dividers producing clutters and more.

\section{Phase Noise Test}

Using a dedicated phase noise tester to measure phase noise can achieve a high sensitivity and accuracy. In the actual work a spectrum analyzer is also often used to measure phase noise, it can directly observe the single sideband phase noise and discrete signal on both sides of the band through a spectrum analyzer. [7]

The Tab.1 is a set of data from actual measurements of phase noise in a certain type of radar receiver.

According to the data in the table, the phase noise of the receiver is mainly influenced by the oscillator. It's obviously that enhancing the local oscillator frequency stability is very important in reducing the phase noise of the channel and improving the signal to noise ratio and receiver sensitivity. 
Table 1 receiver phase noise test

\begin{tabular}{cccc}
\hline Deviation $[\mathrm{Hz}]$ & index $[\mathrm{dBc}]$ & Measured $[\mathrm{dBc}]$ & LO $[\mathrm{dBc}]$ \\
\hline 10 & -60 & -63.1 & -67.5 \\
100 & -75 & -81.5 & -85.7 \\
$1 \mathrm{~K}$ & -85 & -93.2 & -97.3 \\
$10 \mathrm{~K}$ & -95 & -107.2 & -111.7 \\
$100 \mathrm{~K}$ & -105 & -121.3 & -125.5 \\
\hline
\end{tabular}

\section{Summary}

Phase noise has a significant impact on the performance of the radar receiver. Analysis on the affecting factors and the composition of channel phase noise has a very important significance in the study of reducing the channel phase noise and improving the performance of the radar system. When efforts are being made to reduce the phase noise of the channel, how to eliminate the influence of phase noise on the useful signal by the subsequent signal processing means will be the direction of the next in-depth study.

\section{References}

[1] Shuting Gao, Hongsheng Liu, Phase noise analysis and its impact on circuit systems, Fire Control Radar Technology. 2 (2003) 58-63

[2] Daotian Yang, Phase noise of the receiver, China Radio. 10 (2006) 63-65

[3] Xueru Dou, Suling Cui, Principle of phase noise measurement with spectrum analyzer, University Science Research. 437-439

[4] Jia Tian, Wei Wang, Pingyan Shi, Impact of local oscillator phase noise on the digital receiver, Space Electronic Technology. 1 (2012) 17-22

[5] Kunzhi Xu, Shoukan Jing, Zhihong Dai, RF channel phase noise analysis, Electron Equality. 9 (2012) 4-16

[6] Xinyang He, Local oscillator phase noise and its impact on receiver performance, Space Electronic Technology, 1 (2003) 4-13

[7] Yuan Fen, Lei Gu, Phase noise and its testing techniques, Science and Technology Information. 7 (2009) 